\title{
Application of mineral phosphorus fertilizer influences rhizosphere chemical and biological characteristics
}

Hang Liu, Zhongming Hao, Yuhan Yuan, Cuilan Li, Jinjing Zhang

\section{Supplemental material}

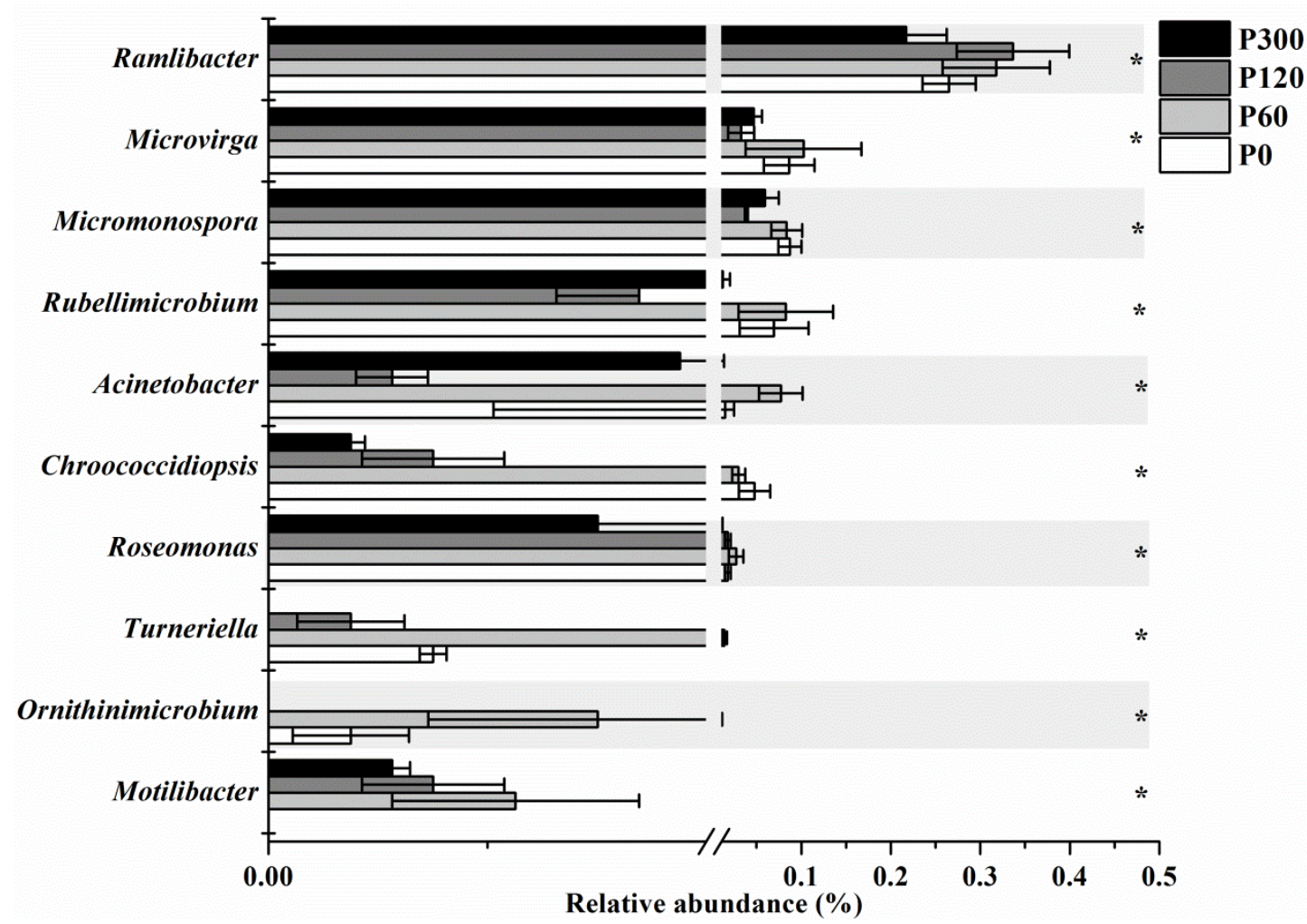

Figure S1. Relative abundances of low-abundance bacterial genera in maize rhizosphere soil under different

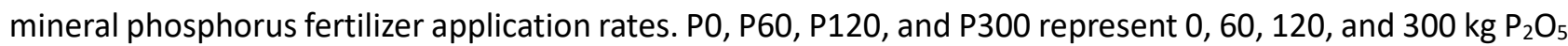
$\mathrm{ha}^{-1}$ of phosphorus fertilizer application rates, respectively. Vertical bars represent standard deviations of the means ( $n=3, * p<0.05$ by Kruskal-Wallis test). 


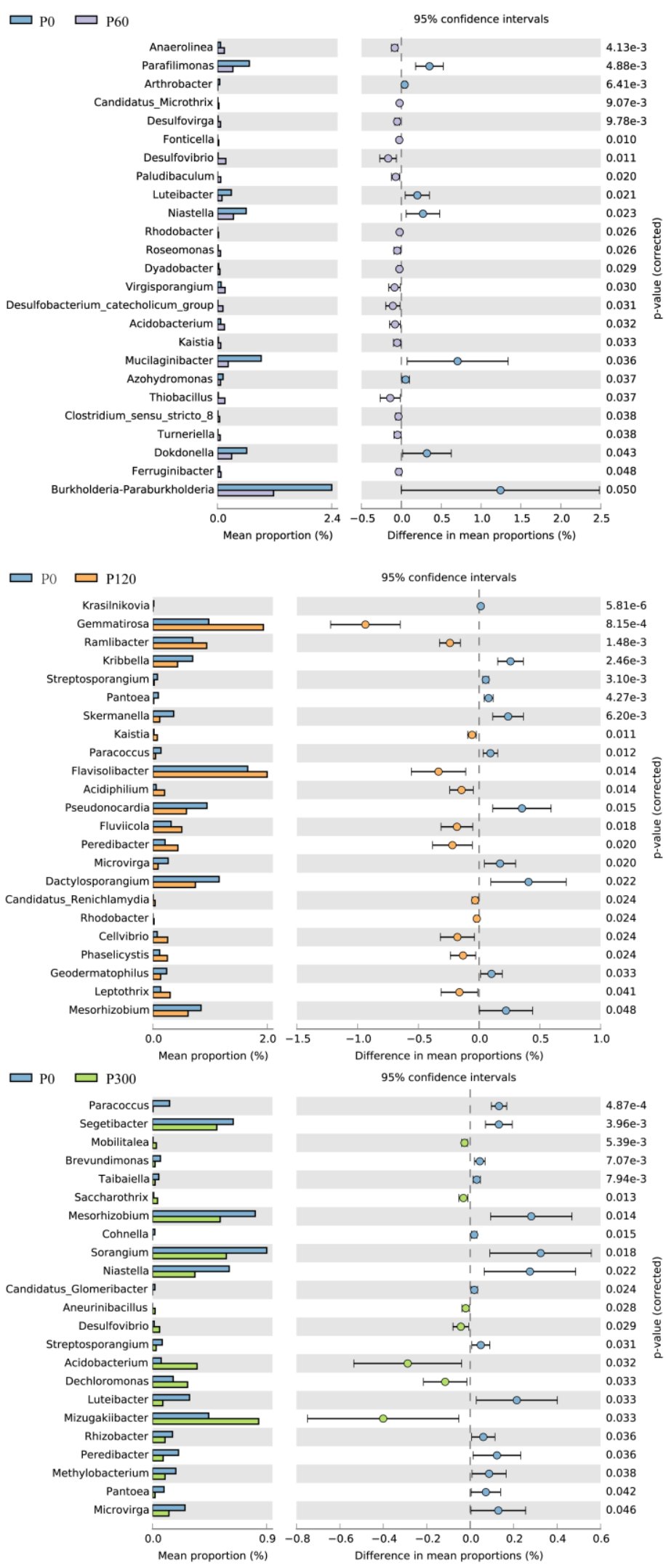

Figure S2. Relative abundances of bacterial genera with significant responses to P0 (at $95 \%$ confidence intervals, Student's t-test, equal variance) in maize rhizosphere soil under different mineral phosphorus fertilizer application rates. $\mathrm{P} 0, \mathrm{P} 60, \mathrm{P} 120$, and $\mathrm{P} 300$ represent $0,60,120$, and $300 \mathrm{~kg} \mathrm{P}_{2} \mathrm{O}_{5} \mathrm{ha}^{-1}$ of phosphorus fertilizer application rates, respectively. 


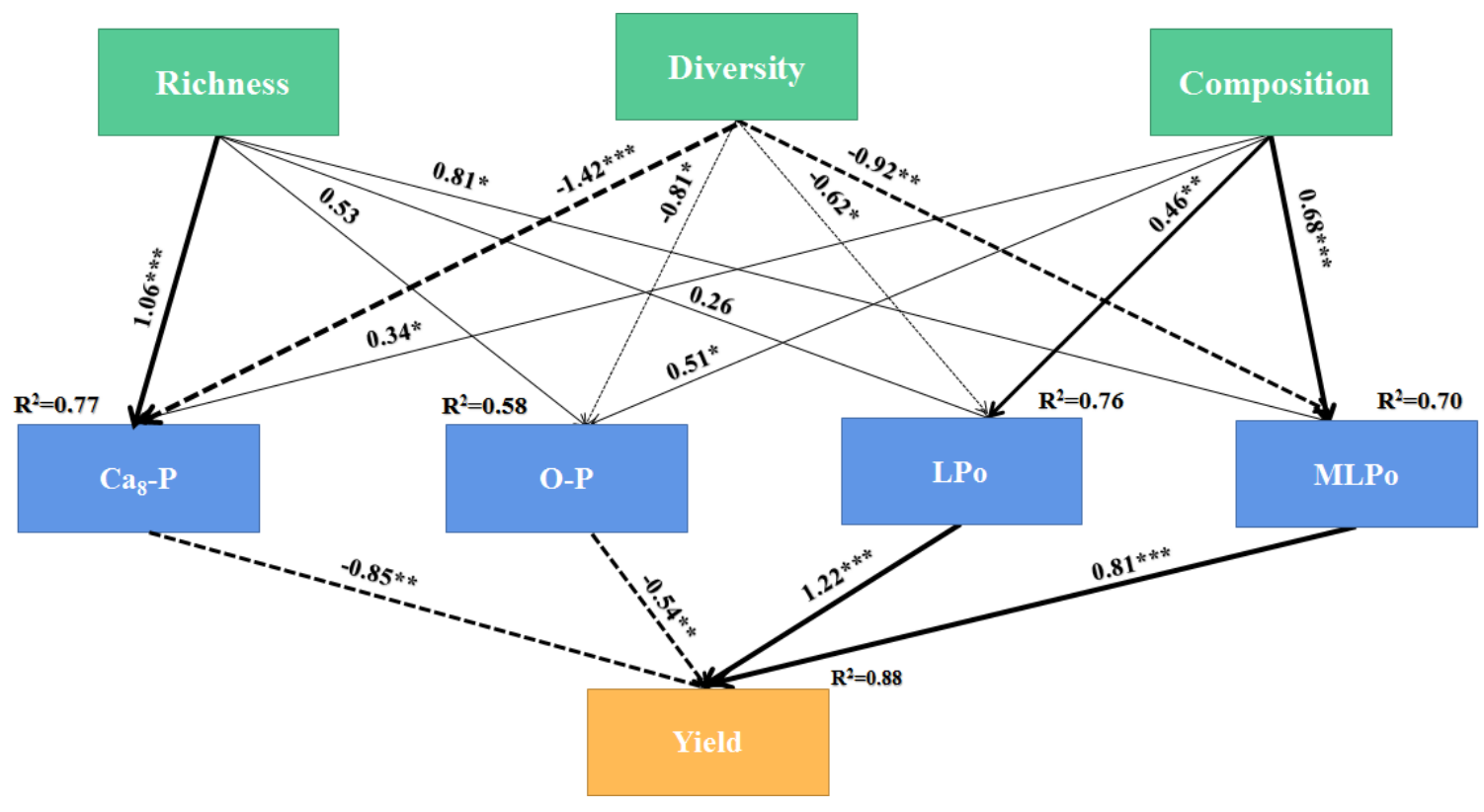

Figure S3. Path diagrams of structural equation modeling (SEM) for the relationship of soil bacterial community diversity and composition on maize yield. Cas-P, octacalcium bound phosphorus; O-P, occluded iron/aluminum bound phosphorus; LPo: labile organic phosphorus; MLPo: moderately labile organic phosphorus. Values above the line represent the path coefficients. Solid lines indicate positive path coefficients and dashed lines indicate negative path coefficients. The width of arrows indicates the strength of the standardized path coefficient $\left({ }^{*} P<0.05,{ }^{* *} P<0.01,{ }^{* * *} P<0.001\right)$. 
Table S1. Soil properties in maize rhizosphere under different mineral phosphorus fertilizer application rates.

\begin{tabular}{|c|c|c|c|c|c|c|c|c|c|c|c|c|c|}
\hline $\begin{array}{l}\text { Treatmen } \\
\mathrm{t}\end{array}$ & $\begin{array}{l}\mathrm{pH} \\
(1: 2.5)\end{array}$ & $\begin{array}{l}\text { SOC } \\
(\mathrm{g} / \mathrm{kg})\end{array}$ & $\begin{array}{l}\text { Olsen P } \\
(\mathrm{mg} / \mathrm{kg})\end{array}$ & $\begin{array}{l}\mathrm{Ca}_{2}-\mathrm{P} \\
(\mathrm{mg} / \mathrm{kg})\end{array}$ & $\begin{array}{l}\mathrm{Ca}_{8}-\mathrm{P} \\
(\mathrm{mg} / \mathrm{kg})\end{array}$ & $\begin{array}{l}\text { Al-P } \\
(\mathrm{mg} / \mathrm{kg})\end{array}$ & $\begin{array}{l}\text { Fe-P } \\
(\mathrm{mg} / \mathrm{kg})\end{array}$ & $\begin{array}{l}\text { O-P } \\
(\mathrm{mg} / \mathrm{kg})\end{array}$ & $\begin{array}{l}\mathrm{Ca}_{10}-\mathrm{P} \\
(\mathrm{mg} / \mathrm{kg})\end{array}$ & $\begin{array}{l}\text { LPo } \\
(\mathrm{mg} / \mathrm{kg})\end{array}$ & $\begin{array}{l}\text { MLPo } \\
(\mathrm{mg} / \mathrm{kg})\end{array}$ & $\begin{array}{l}\text { MRPo } \\
(\mathrm{mg} / \mathrm{kg})\end{array}$ & $\begin{array}{l}\text { HRPo } \\
(\mathrm{mg} / \mathrm{kg})\end{array}$ \\
\hline PO & $6.51 \pm 0.39 a$ & $20.1 \pm 0.49 a$ & $31.9 \pm 0.27 d$ & $17.1 \pm 3.18 \mathrm{c}$ & $8.24 \pm 1.27 b$ & $24.6 \pm 3.49 \mathrm{~b}$ & $52.9 \pm 5.04 a$ & $48.3 \pm 3.48 b$ & $69.1 \pm 4.22 c$ & $14.2 \pm 1.97 \mathrm{~b}$ & $46.0 \pm 6.09 \mathrm{~b}$ & $8.20 \pm 4.80 c$ & $15.5 \pm 3.31 a$ \\
\hline P60 & $6.25 \pm 0.32 a$ & $19.3 \pm 0.78 a b$ & $45.7 \pm 1.13 c$ & $20.0 \pm 3.05 b c$ & $9.76 \pm 2.85 b$ & $26.5 \pm 6.18 b$ & $54.4 \pm 3.59 a$ & $51.8 \pm 4.93 b$ & $75.3 \pm 9.15 c$ & $17.8 \pm 1.58 \mathrm{~b}$ & $63.6 \pm 7.45 a$ & $17.0 \pm 6.84 b c$ & $23.2 \pm 5.53 a$ \\
\hline P120 & $6.15 \pm 0.32 a$ & $18.8 \pm 0.48 b$ & $52.3 \pm 1.02 b$ & $21.3 \pm 1.69 b$ & $14.3 \pm 1.00 \mathrm{a}$ & $33.2 \pm 4.50 \mathrm{~b}$ & $54.5 \pm 2.11 a$ & $51.2 \pm 3.74 b$ & $91.4 \pm 13.3 \mathrm{~b}$ & $23.3 \pm 2.22 \mathrm{a}$ & $71.6 \pm 3.61 a$ & $27.3 \pm 10.4 a b$ & $26.1 \pm 14.6 a$ \\
\hline P300 & $6.24 \pm 0.43 a$ & $18.5 \pm 0.68 b$ & $76.7 \pm 1.30 \mathrm{a}$ & $31.8 \pm 2.06 a$ & $14.5 \pm 4.06 a$ & $43.7 \pm 4.87 a$ & $60.6 \pm 13.9 a$ & $68.2 \pm 8.60 \mathrm{a}$ & $107.7 \pm 12.2 \mathrm{a}$ & $25.2 \pm 4.41 a$ & $74.5 \pm 16.3 a$ & $31.9 \pm 4.12 \mathrm{a}$ & $27.0 \pm 5.26 a$ \\
\hline
\end{tabular}

Values followed by different lowercase letters within the same column are significantly different at $P<0.05$ determined by least significant difference (LSD).

P0, P60, P120, and P300 represent 0,60, 120, and $300 \mathrm{~kg} \mathrm{P}_{2} \mathrm{O}_{5}$ ha-1 of phosphorus fertilizer application rates, respectively. SOC, soil organic carbon; Ca $-\mathrm{P}$, dicalcium bound phosphorus; Ca $\mathrm{P}^{-\mathrm{P}}$, octacalcium bound phosphorus; Al-P, aluminum bound phosphorus; Fe-P, iron bound phosphorus; O-P, occluded iron/aluminum bound phosphorus; Ca 10 -P: phosphorite bound phosphorus; LPo: labile organic phosphorus; MLPo: moderately labile organic phosphorus; MRPo: moderately resistant organic phosphorus; HRPo: highly resistant organic phosphorus.

Table S2. Phosphorus use efficiency, maize grain yield, and yield componentsunder different mineral phosphorus fertilizer application rates.

\begin{tabular}{|c|c|c|c|c|c|c|c|c|c|c|}
\hline Treatment & $\operatorname{PFP}\left(\mathrm{kg} \mathrm{kg}^{-1}\right)$ & PAE $\left(\mathrm{kg} \mathrm{kg}^{-1}\right)$ & PAUE (\%) & PUPE $\left(\mathrm{kg} \mathrm{kg}^{-1}\right)$ & $\mathrm{EL}(\mathrm{cm})$ & BTL $(\mathrm{cm})$ & ERN & KNR & HKW (g) & Yield $\left(\mathrm{g} \mathrm{pot}^{-1}\right)$ \\
\hline PO & -- & -- & -- & -- & $14.7 \pm 0.33 b$ & $1.40 \pm 0.73 a$ & $16.3 \pm 1.51 \mathrm{a}$ & $30.5 \pm 2.43 b$ & $27.8 \pm 2.07 a$ & $109.9 \pm 7.15 b$ \\
\hline P60 & $136.9 \pm 3.00 \mathrm{a}$ & $17.8 \pm 9.48 a$ & $39.2 \pm 14.9 a$ & $1.40 \pm 0.08 a$ & $15.5 \pm 0.42 \mathrm{a}$ & $0.87 \pm 0.92 \mathrm{a}$ & $16.0 \pm 1.79 a$ & $32.0 \pm 2.19 a b$ & $29.8 \pm 2.15 a$ & $126.4 \pm 2.77 \mathrm{a}$ \\
\hline P120 & $64.9 \pm 4.29 b$ & $5.39 \pm 2.95 b$ & $27.9 \pm 4.62 \mathrm{a}$ & $0.78 \pm 0.04 b$ & $14.5 \pm 0.61 b$ & $0.90 \pm 0.99 a$ & $15.0 \pm 1.10 \mathrm{ab}$ & $30.3 \pm 1.75 b$ & $29.6 \pm 1.20 \mathrm{a}$ & $126.0 \pm 5.74 \mathrm{a}$ \\
\hline P300 & $27.3 \pm 1.77 c$ & $3.49 \pm 0.95 b$ & $11.6 \pm 1.80 \mathrm{~b}$ & $0.32 \pm 0.01 \mathrm{c}$ & $15.8 \pm 0.67 a$ & $0.42 \pm 0.65 a$ & $14.3 \pm 0.82 b$ & $34.5 \pm 2.26 \mathrm{a}$ & $29.6 \pm 2.97 \mathrm{a}$ & $119.9 \pm 2.62 \mathrm{a}$ \\
\hline
\end{tabular}

P0, P60, P120, and P300 represent 0,60, 120, and $300 \mathrm{~kg} \mathrm{P}_{2} \mathrm{O}_{5}$ ha-1 of phosphorus fertilizer application rates, respectively. PFP: partial factor productivity; PAE: phosphorus agronomic efficiency; PAUE: phosphorus apparent utilization efficiency; PUPE: phosphorus uptake efficiency; EL, ear length; BTL, bald tip length; ERN, ear row number; KNR, kernel number per row; HKW, hundred kernel weight;. 
Table S3. Illumina MiSeq sequenced bacterial data (at 97\% sequence similarity) based on the $16 \mathrm{~S}$ rRNA gene under different mineral phosphorus fertilizer application rates.

\begin{tabular}{llllll}
\hline Treatment & Sequences & Bases (bp) & Average length (bp) & Maximum length (bp) & Minimum length (bp) \\
\hline P0-1 & 137087 & 57054428 & 416 & 450 & 249 \\
P0-2 & 97211 & 40375348 & 415 & 449 & 258 \\
P0-3 & 42821 & 17788881 & 415 & 447 & 386 \\
P60-1 & 99521 & 41395044 & 416 & 447 & 237 \\
P60-2 & 45731 & 19060343 & 417 & 443 & 310 \\
P60-3 & 96905 & 40466087 & 418 & 444 & 276 \\
P120-1 & 53845 & 22392341 & 416 & 443 & 318 \\
P120-2 & 110243 & 46006717 & 417 & 450 & 262 \\
P120-3 & 49309 & 20495173 & 416 & 449 & 262 \\
P300-1 & 37227 & 15478750 & 416 & 447 & 262 \\
P300-2 & 116993 & 48644604 & 416 & 447 & 276 \\
P300-3 & 76923 & 31950732 & 415 & 443 & 239 \\
\hline
\end{tabular}

P0, P60, P120, and P300 represent $0,60,120$, and $300 \mathrm{~kg} \mathrm{P}_{2} \mathrm{O}_{5}$ ha $^{-1}$ of phosphorus fertilizer application rates, respectively.

Table S4. Relative abundance (\%) at bacterial phyla level under different mineral phosphorus fertilizer application rates.

\begin{tabular}{|c|c|c|c|c|c|c|}
\hline Treatment & Chloroflexi & Firmicutes & Nitrospirae & Cyanobacteria & $\begin{array}{l}\text { Gemmatimonadete } \\
\mathrm{s}\end{array}$ & Bacteroidetes \\
\hline PO & 9.86 & 1.60 & 0.38 & 1.37 & 4.22 & 4.52 \\
\hline P60 & 10.5 & 1.33 & 0.43 & 1.01 & 5.40 & 3.85 \\
\hline P120 & 7.57 & 1.75 & 0.56 & 0.66 & 6.23 & 4.71 \\
\hline P300 & 9.48 & 1.51 & 0.55 & 0.65 & 5.14 & 3.73 \\
\hline \multicolumn{7}{|l|}{$P$ value } \\
\hline P0 vs P60 & ns & $\mathrm{ns}$ & ns & ns & 0.034 & $5.41 \mathrm{E}-03$ \\
\hline $\mathrm{P0}$ vs P120 & 0.018 & ns & ns & ns & $1.74 \mathrm{E}-03$ & ns \\
\hline P0 vs P300 & 0.029 & ns & ns & ns & $2.96 \mathrm{E}-03$ & 0.014 \\
\hline
\end{tabular}

$\mathrm{P0}, \mathrm{P} 60, \mathrm{P} 120$, and $\mathrm{P} 300$ represent $0,60,120$, and $300 \mathrm{~kg} \mathrm{P}_{2} \mathrm{O}_{5}$ ha-1 of phosphorus fertilizer application rates, respectively. 
Table S5. Indicator species in maize rhizosphere soil under different mineral phosphorus fertilizer application rates.

\begin{tabular}{|c|c|c|c|c|c|c|c|c|c|c|c|}
\hline Treatment & Indicator species ${ }^{a}$ & $A^{b}$ & $\mathrm{~B}^{\mathrm{b}}$ & stat $^{\mathrm{b}}$ & IndVal ${ }^{b}$ & $p$-value ${ }^{c}$ & Taxonomy ${ }^{d}$ & Identities (\%) & Bit score & E-value & Accession No. \\
\hline \multirow[t]{10}{*}{ PO } & OTU3501 & 1 & 1 & 1.000 & 1.0000 & 0.021 & g_Brachybacterium & 100.00 & 409 & 0.0 & KX645681.1 \\
\hline & OTU3097 & 0.9091 & 1 & 0.953 & 0.9091 & 0.035 & g_CandidatusMoeniiplasma & 91.10 & 309 & $8 \mathrm{E}-159$ & KP763348.1 \\
\hline & OTU1462 & 0.8182 & 1 & 0.905 & 0.8182 & 0.039 & g_Pseudomonas & 99.53 & 423 & 0.0 & MN519642.1 \\
\hline & OTU580 & 0.7778 & 1 & 0.882 & 0.7778 & 0.021 & o_Cystobacterineae & 93.26 & 343 & $1 \mathrm{E}-177$ & KX367460.1 \\
\hline & OTU291 & 0.7500 & 1 & 0.866 & 0.75 & 0.021 & o_unculturedDesulfuromonadales & 97.67 & 400 & 0.0 & LN555055.1 \\
\hline & OTU761 & 0.7321 & 1 & 0.856 & 0.7321 & 0.021 & s_Paraburkholderia sp. & 99.77 & 426 & 0.0 & MH925968.1 \\
\hline & OTU4239 & 0.6667 & 1 & 0.816 & 0.6667 & 0.039 & p_candidatusSaccharibacteria & 93.28 & 321 & $2 \mathrm{E}-165$ & KJ191955.1 \\
\hline & OTU807 & 0.6081 & 1 & 0.780 & 0.6081 & 0.037 & c_unculturedGammaproteobacterium & 95.34 & 369 & 0.0 & EF074577.1 \\
\hline & OTU2486 & 0.5811 & 1 & 0.762 & 0.5811 & 0.021 & s_Niastella sp. & 97.41 & 391 & 0.0 & EU917053.1 \\
\hline & OTU4429 & 0.5455 & 1 & 0.739 & 0.5455 & 0.021 & o_unculturedMyxococcales & 98.27 & 384 & 0.0 & KX367290.1 \\
\hline \multirow[t]{10}{*}{ P60 } & OTU2173 & 1.0000 & 1 & 1.000 & 1.0000 & 0.019 & s__unculturedBlastopirellula sp. & 95.32 & 367 & 0.0 & JN832629.1 \\
\hline & OTU5171 & 0.8049 & 1 & 0.897 & 0.8049 & 0.042 & $\mathrm{~s} \_$Magnetospirillum sp. & 98.76 & 389 & 0.0 & MH686102.1 \\
\hline & OTU3327 & 0.8571 & 1 & 0.926 & 0.8571 & 0.037 & s__Ornithinimicrobiumkibberense & 100.00 & 411 & 0.0 & MK726118.1 \\
\hline & OTU2623 & 0.6923 & 1 & 0.832 & 0.6923 & 0.042 & o__unculturedMyxococcales & 97.64 & 394 & 0.0 & FJ889280.1 \\
\hline & OTU4281 & 0.6923 & 1 & 0.832 & 0.6923 & 0.035 & p_unculturedGemmatimonadetes & 95.56 & 351 & 0.0 & JN002780.1 \\
\hline & OTU3714 & 0.5778 & 1 & 0.760 & 0.5778 & 0.019 & p_unculturedChloroflexi & 95.82 & 354 & 0.0 & KF183051.1 \\
\hline & OTU2275 & 0.5664 & 1 & 0.753 & 0.5664 & 0.019 & s__unculturedElusimicrobium sp. & 96.24 & 376 & 0.0 & GQ375494.1 \\
\hline & OTU3296 & 0.5556 & 1 & 0.745 & 0.5556 & 0.019 & g_unculturedClostridia & 93.70 & 333 & $3 E-172$ & JQ700615.1 \\
\hline & OTU3598 & 0.5517 & 1 & 0.743 & 0.5517 & 0.019 & s_unculturedTychonema sp. & 98.28 & 386 & 0.0 & EU809762.1 \\
\hline & OTU84 & 0.5040 & 1 & 0.710 & 0.5040 & 0.019 & s__unculturedGeobacter sp. & 100.00 & 431 & 0.0 & JN091600.1 \\
\hline
\end{tabular}


Table S5. (Continue).

\begin{tabular}{|c|c|c|c|c|c|c|c|c|c|c|c|}
\hline \multirow[t]{5}{*}{ P120 } & OTU2267 & 0.7664 & 1 & 0.875 & 0.7664 & 0.044 & s_unculturedCroceibacter sp. & 92.24 & 324 & $3 E-167$ & GU245904.1 \\
\hline & OTU855 & 0.6939 & 1 & 0.833 & 0.6939 & 0.015 & c_unculturedGammaproteobacterium & 92.79 & 336 & $8 \mathrm{E}-174$ & EF074555.1 \\
\hline & OTU803 & 0.5926 & 1 & 0.770 & 0.5926 & 0.026 & s__Delftiatsuruhatensis & 100.00 & 429 & 0.0 & MN393973.1 \\
\hline & OTU4027 & 0.5400 & 1 & 0.735 & 0.5400 & 0.044 & s__CandidatusSaccharimonas sp. & 97.77 & 377 & 0.0 & KX028761.1 \\
\hline & OTU1758 & 0.5161 & 1 & 0.718 & 0.5161 & 0.026 & c_unculturedGammaproteobacterium & 97.90 & 401 & 0.0 & GQ302552.1 \\
\hline \multirow[t]{10}{*}{ P300 } & OTU1888 & 0.8750 & 1 & 0.935 & 0.8750 & 0.015 & s__Paenibacillus sp. & 94.87 & 362 & 0.0 & KY684255.2 \\
\hline & OTU4481 & 0.8235 & 1 & 0.907 & 0.8235 & 0.015 & s_unculturedLongilinea sp. & 95.31 & 347 & $5 \mathrm{E}-180$ & GQ421109.1 \\
\hline & OTU4315 & 0.8182 & 1 & 0.905 & 0.8182 & 0.015 & p_unculturedCandidatusSaccharibacteria & 94.10 & 333 & $3 E-172$ & MF439312.1 \\
\hline & OTU1928 & 0.6364 & 1 & 0.798 & 0.6364 & 0.015 & s_unculturedGeobacter sp. & 97.20 & 391 & 0.0 & AM941477.1 \\
\hline & OTU2747 & 0.6176 & 1 & 0.786 & 0.6176 & 0.048 & s_unculturedSporocytophaga sp. & 94.81 & 357 & 0.0 & MH174164.1 \\
\hline & OTU3584 & 0.6163 & 1 & 0.785 & 0.6163 & 0.015 & s_unculturedPhormidium sp. & 95.58 & 353 & 0.0 & GQ421006.1 \\
\hline & OTU4151 & 0.5862 & 1 & 0.766 & 0.5862 & 0.015 & p_unculturedCandidatusSaccharibacteria & 95.33 & 348 & $2 \mathrm{E}-180$ & LC381003.1 \\
\hline & OTU4094 & 0.5706 & 1 & 0.755 & 0.5706 & 0.015 & p_unculturedCandidatusSaccharibacteria & 95.25 & 323 & $1 E-166$ & KT271006.1 \\
\hline & OTU4879 & 0.5200 & 1 & 0.721 & 0.5200 & 0.015 & s__unculturedAcidobacterium sp. & 98.51 & 386 & 0.0 & KF035826.1 \\
\hline & OTU2966 & 0.5075 & 1 & 0.712 & 0.5075 & 0.015 & p__Gemmatimonadetes & 96.44 & 376 & 0.0 & AY673312.1 \\
\hline
\end{tabular}

P0, P60, P120, and P300 represent 0,60,120, and $300 \mathrm{~kg} \mathrm{P}_{2} \mathrm{O}_{5}$ ha $^{-1}$ of phosphorus fertilizer application rates, respectively.

a OTUs with IndVal > 0.5 were selected.

${ }^{b} \mathrm{~A}$ is called specificity or probable predictive value, i.e. if $\mathrm{A}=1$ for species calpurp, then calpurp is a good indicator because it occurs only in that group $B$ is called fidelity or sensitivity, i.e. if $B=1$ for species calpurp, then all sites within the group contain calpurp. Stat is the average of $A$ and $B$. IndVal= $A^{*} B^{*}$, IndVal $>0.5$ were selected for analyze.

${ }^{c} p$-value is based on 1000 Monte Carlo permutations.

d Taxonomy of OTUs were identified by using blastn in NCBI: $p$, phylum; c, class; $o$, order; g, genus; s, species. 
Table S6. Correlation coefficients between soil properties and bacterial community.

\begin{tabular}{|c|c|c|c|c|c|c|c|c|c|c|c|c|c|c|c|c|c|}
\hline & $\mathrm{pH}$ & SOC & $\begin{array}{l}\text { Olsen } \\
\mathrm{P}\end{array}$ & $\mathrm{Ca}_{2}-\mathrm{P}$ & $\mathrm{Ca}_{8}-\mathrm{P}$ & Al-P & Fe-P & O-P & $\mathrm{Ca}_{10}-\mathrm{P}$ & LPo & MLPo & MRPo & HRPo & Shannon & Chao & Comp1 & Comp2 \\
\hline \multirow[t]{2}{*}{$\mathrm{pH}$} & 1.00 & $0.69^{*}$ & $-0.58^{*}$ & -0.42 & $-0.69 *$ & $-0.62^{*}$ & -0.24 & $-0.65^{*}$ & -0.37 & - & $-0.74^{* *}$ & $-0.61^{*}$ & -0.49 & $0.63^{*}$ & 0.57 & 0.27 & - \\
\hline & & & & & & & & & & $0.89^{* * *}$ & & & & & & & $0.91^{* * *}$ \\
\hline SOC & & 1.00 & $-0.61^{*}$ & $-0.58^{*}$ & -0.56 & $-0.67^{*}$ & -0.15 & $-0.77^{* *}$ & $-0.69^{*}$ & $-0.68^{*}$ & $-0.67^{*}$ & $-0.69^{*}$ & -0.43 & 0.52 & 0.39 & 0.39 & $-0.66^{*}$ \\
\hline Olsen P & & & 1.00 & $0.91^{* * *}$ & $0.63^{*}$ & $0.91^{* * *}$ & $0.64^{*}$ & $0.90^{* * *}$ & $0.84^{* * *}$ & $0.74^{* *}$ & 0.51 & $0.81^{* *}$ & 0.55 & $-0.62^{*}$ & -0.53 & $-0.60^{*}$ & $0.59 *$ \\
\hline $\mathrm{Ca}_{2}-\mathrm{P}$ & & & & 1.00 & 0.50 & $0.87^{* * *}$ & 0.57 & $0.86^{* *}$ & $0.85^{* * *}$ & 0.56 & 0.49 & $0.81^{* *}$ & 0.49 & -0.38 & -0.31 & -0.45 & 0.53 \\
\hline $\mathrm{Ca}_{8}-\mathrm{P}$ & & & & & 1.00 & $0.76^{* *}$ & 0.30 & $0.78^{* *}$ & 0.52 & $0.82^{* *}$ & $0.78^{* *}$ & $0.59^{*}$ & 0.24 & $-0.66^{*}$ & -0.31 & $-0.69 *$ & 0.49 \\
\hline Al-P & & & & & & 1.00 & 0.48 & $0.93^{* * *}$ & $0.87^{* * *}$ & $0.77^{* *}$ & $0.66^{*}$ & $0.88^{* * *}$ & $0.59 *$ & $-0.61^{*}$ & -0.42 & $-0.59^{*}$ & $0.62^{*}$ \\
\hline Fe-P & & & & & & & 1.00 & 0.46 & 0.39 & 0.35 & 0.06 & 0.40 & 0.50 & 0.01 & 0.03 & -0.21 & 0.26 \\
\hline O-P & & & & & & & & 1.00 & $0.78^{* *}$ & $0.77^{* *}$ & $0.68^{*}$ & $0.83^{* * *}$ & 0.41 & -0.58 & -0.39 & $-0.61^{*}$ & $0.63^{*}$ \\
\hline $\mathrm{Ca}_{10}-\mathrm{P}$ & & & & & & & & & 1.00 & 0.56 & 0.57 & $0.75^{* *}$ & $0.59 *$ & -0.55 & -0.41 & -0.56 & 0.41 \\
\hline LPo & & & & & & & & & & 1.00 & $0.68^{*}$ & $0.75^{* *}$ & 0.51 & $-0.77^{* *}$ & $-0.67^{*}$ & $-0.65^{*}$ & $0.71^{*}$ \\
\hline MLPo & & & & & & & & & & & 1.00 & 0.47 & 0.34 & -0.51 & -0.27 & -0.27 & $0.69^{*}$ \\
\hline MRPo & & & & & & & & & & & & 1.00 & 0.46 & -0.52 & -0.40 & -0.55 & $0.61^{*}$ \\
\hline HRPo & & & & & & & & & & & & & 1.00 & -0.25 & -0.38 & -0.09 & 0.54 \\
\hline Shannon & & & & & & & & & & & & & & 1.00 & $0.86^{* * *}$ & $0.74^{* *}$ & -0.44 \\
\hline Chao & & & & & & & & & & & & & & & 1.00 & 0.52 & -0.44 \\
\hline Comp1 & & & & & & & & & & & & & & & & 1.00 & $<0.001$ \\
\hline Comp2 & & & & & & & & & & & & & & & & & 1.00 \\
\hline
\end{tabular}

${ }^{*} P<0.05,{ }^{* *} P<0.01,{ }^{* * *} P<0.001$

SOC, soil organic carbon; $\mathrm{Ca}_{2}-\mathrm{P}$, dicalcium bound phosphorus; Сав $-\mathrm{P}$, octacalcium bound phosphorus; Al-P, aluminum bound phosphorus; Fe-P, iron bound phosphorus; O-P, occluded iron/aluminum bound phosphorus; $\mathrm{Ca}_{10} \mathrm{P}$ : phosphorite bound phosphorus; LPo: labile organic phosphorus; MLPo: moderately labile organic phosphorus; MRPo: moderately resistant organic phosphorus; HRPo: highly resistant organic phosphorus; Comp: component. 
Table S7. Variation portioning into soil bacterial community in maize rhizosphere soil under different mineral phosphorus fertilizer application rates explained by soil properties.

\begin{tabular}{llll}
\hline Soil properties & Contribution\% & Pseudo-F & $P$ \\
\hline LPo & 24.6 & 3.3 & 0.010 \\
$\mathrm{pH}$ & 12.1 & 1.7 & 0.136 \\
$\mathrm{Fe}-\mathrm{P}$ & 11.1 & 1.7 & 0.132 \\
$\mathrm{Ca} \mathrm{a}_{2}-\mathrm{P}$ & 9.8 & 1.9 & 0.124 \\
Olsen P & 9.2 & 1.5 & 0.176 \\
O-P & 6.9 & 1.1 & 0.418 \\
MLPo & 6.7 & 1.9 & 0.326 \\
SOC & 5.9 & 1.2 & 0.370 \\
Ca 8 -P & 5.6 & 1.1 & 0.392 \\
Ca ${ }_{10}-\mathrm{P}$ & 4.7 & 0.9 & 0.466 \\
HRPo & 3.4 & $<0.1$ & 1.000 \\
\hline
\end{tabular}

LPo: labile organic phosphorus; Fe-P, iron bound phosphorus; $\mathrm{Ca}_{2}-\mathrm{P}$, dicalcium bound phosphorus; O-P, occluded iron/aluminum bound phosphorus; MLPo: moderately labile organic phosphorus; SOC, soil organic carbon; $\mathrm{Ca}_{8}-\mathrm{P}$, octacalcium bound phosphorus; $\mathrm{Ca}_{10}-\mathrm{P}$ : phosphorite bound phosphorus; HRPo: highly resistant organic phosphorus. 